\title{
Pyruvate enhances M1 microglial polarization via NF-KB/MCT1/iNOS signaling axis under low glucose condition
}

\author{
Peng Zhou \\ institute of neuroscience $\varangle$ Basic Medical College of Wenzhou Medical University \\ Juan-juan Du \\ institute of neuroscience $₫$ Basic Medical College of Wenzhou Medical University \\ Tao Zhou \\ institute of neuroscience $₫$ Basic Medical College of Wenzhou Medical University \\ Chen-you Sun \\ institute of neuroscience $\mathbb{B}$ Basic Medical College of Wenzhou Medical University \\ Huai-rui Cui \\ Department of Anatomy $₫$ Basic Medical College of Wenzhou Medical University \\ Mao-chao Ding \\ Department of Anatomy $₫$ Basic Medical College of Wenzhou Medical University \\ Chao-xian Yang

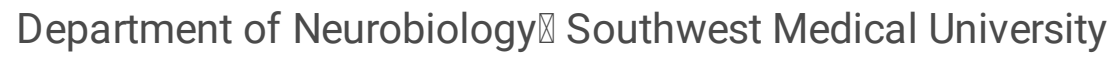 \\ Min Liao ( $D$ liaominzp@163.com ) \\ wenzhou medical university https://orcid.org/0000-0002-6141-1626
}

\section{Research}

Keywords: Microglia, INOS, MCT1, Pyruvate, Ischemia, Low glucose condition

Posted Date: June 8th, 2020

DOI: https://doi.org/10.21203/rs.3.rs-33328/v1

License: (c) (1) This work is licensed under a Creative Commons Attribution 4.0 International License. Read Full License 


\section{Abstract \\ Background}

Proinflammatory microglia rely predominantly on glycolysis to maintain cytokine production during an inflammatory response. However, during ischemia, where glucose supply is low, inducible nitric oxide synthase (iNOS) production remains high accompanied by an increase in monocarboxylate transporter 1 (MCT1) expression. In this study, we explored whether there is a link between iNOS and MCT1 expressions, and whether pyruvate can act as an energy source to sustain the M1 phenotype.

\section{Methods}

Using a mouse model with laser-induced brain ischemia and cell culture with low-glucose treatment, we examined responses from microglia.

\section{Results}

The expressions of iNOS and MCT1, as well as arginase-1 (ARG1), were increased in the brain of the ischemic mouse model. In the BV2 microglial cell line and primary microglia treated under low glucose condition, iNOS and MCT1 also increased, while ARG1 decreased. The addition of pyruvate under lowglucose or lipopolysaccharide (LPS) treatment enhanced iNOS and MCT1 expressions compared with groups without pyruvate. MCT1 knockdown resulted in decreased iNOS while MCT1 overexpression increased iNOS. Furthermore, inhibitor of nuclear factor-kappaB (NF-KB) reduced both iNOS and MCT1.

\section{Conclusion}

Our data suggested that after proinflammatory microglial polarization, MCT1 is upregulated through the NF-KB signaling pathway, which leads to iNOS production. We speculate that microglia may continuously pick up monocarboxylates such as pyruvate through MCT1 to sustain the M1 phenotype.

\section{Introduction}

Microglia, resident immune cells in the central nervous system (CNS), originate from the yolk sac progenitors. They survey the microenvironment and respond quickly to events from microbial invasion[1], trauma[2], chronic neurodegenerative diseases[3-6] to a variety of signaling[7]. A multitude of functions have been assigned to microglia[8], including secretion of pro-inflammatory cytokines, phagocytosis of cellular debris[9, 10], support of neuronal growth[11-13] and re-myelination[14-16]. M1 microglia initiate the inflammatory response in early stages of injury $[17,18]$ and express marker proteins like inducible nitric oxide synthase (iNOS), CD16/32 and CD14; whereas, the M2 microglia express arginase-1 (ARG1), CD206 and Y1 and carry out tissue repair during the later stage of injury[19-21]. The availability of 
energy substrates is vital to quiescent and activated microglia[22, 23]. Oxygen-glucose deprivation boosts microglial phagocytosis and the release of inflammatory cytokines[24]. Shortage of glucose promotes pro-inflammatory cytokine release through a mechanism related to the NADH:NAD ratio in the cytoplasm[25]. In spite of the variation in microglial phenotypes, they all need an orchestrated pathway to regulate ATP production[26]. It is well established that anaerobic glycolysis, which produces pyruvate, is the predominant way for adenosine triphosphate (ATP) production in the M1 phenotype, while the M2 phenotype rely more on the tricarboxylic acid cycle[27, 28]. However, the post-injury niche is one with low glucose and high lactate, which could not sustain the glycolytic metabolism during M1 microglial polarization. We know through experimental evidence that microglia indeed polarize to and stay in the M1 phenotype in vivo after injuries[21, 29-31] and a large amount of pyruvate produced by glycolysis within the cell is converted to lactate, both of which are monocarboxylates.

Monocarboxylate transporter 1(MCT1) is an enzyme that catalyzes the transfer of lactate, pyruvate and other monocarboxylates across the cellular membrane and inner mitochondrial membrane[32, 33]. MCT1 expression was found to be upregulated in microglia after CNS injury [34]and after M1 polarization [3540]. MCT1 was also shown to drive tumor angiogenesis through the nuclear factor-kappaB (NF-KB) pathway[28, 41]. NF-KB is a well-established master transcription factor of pro-inflammatory markers in microglia, including the upregulation of iNOS, IL-1, IL-6 and TNF-a[42]. In particular, high level of iNOS is crucial for phagocytosis in microglia[43, 44]. In this study, we hypothesized that within ischemic surroundings, microglia continuously fuel the mitochondria through picking up pyruvate to sustain the M1 phenotype and that pyruvate may be taken up by MCT1 and utilized to sustain M1 polarization in microglia. We utilized in vivo and in vitro methods to test whether M1 microglial polarization affected MCT1 expression and whether iNOS expression is changed during MCT1 over-expression and knockdown. Furthermore, we asked whether pyruvate can act as an alternative energy substrate to sustain the M1 phenotype.

\section{Materials And Methods}

\section{Animals}

All animals used in this experiment were in compliance with guidelines from Animal Experiment Ethical Inspection of Laboratory Animal Centre at Wenzhou Medical University. Eight-week-old male and female mice were housed in standard cages with $12 \mathrm{~h}$ dark/light cycle at $23 \pm 1^{\circ} \mathrm{C}$ and $50 \pm 5 \%$ humidity and supplied with food and water ad libitum.

\section{Laser-induced ischemia model in the brain and TTC staining}

The laser-induced ischemia model in mice was described in detail from a previous publication [39]. Briefly, C57BL/ 6 mice were first injected with rose bengal stain intraperitoneally. Then, mouse head was fixed on a three-dimensional brain locator and opened at the sagittal line after general anesthesia $(50 \mathrm{mg} / \mathrm{kg}$ ) by intraperitoneal injection of $1 \%$ Pentobarbital sodium. A hole was created in the calvarium 
(2.0 $\mathrm{mm}$ left of the sagittal line and $1.0 \mathrm{~mm}$ in front of the coronal line), and an optic fiber was inserted to a depth of $3.0 \mathrm{~mm}$ where the brain was irradiated with the $473 \mathrm{~nm}$ blue laser for $15 \mathrm{~min}$. Mice were then sutured up and monitored on a heating plate. After 24 hours, the mice were sacrificed and the brains were stripped off the cerebellum, olfactory bulb and lower brainstem, and placed in $4{ }^{\circ} \mathrm{C} P B S$ solution. Then, the brain was left frozen at $-20^{\circ} \mathrm{C}$ for $30 \mathrm{~min}$, and cut into slices with a thickness of $1 \mathrm{~mm}$. The slices were immersed in $2 \%$ 2,3,5-triphenyl tetrazolium chloride (TTC) solution in a $37^{\circ} \mathrm{C}$ water bath shaker for 30 min. Afterwards, the slices were washed in PBS solution for 5 min and photographed.

\section{Primary cell culture and treatments}

Microglia were isolated from 1-day-old postnatal mouse based on established protocol[45]. Briefly, cortical cells were acquired from the pups sacrificed by cervical dislocation. Cells were cultured in DMEM/F12 supplemented with $10 \%$ fetal bovine serum and $0.5 \mathrm{ng} / \mathrm{ml}$ of granulocyte-macrophage colony-stimulating factor (GM-CSF). At the end of 10 days, microglia were sub-cultured into 6-well or 24well plates at a density of $5 \times 10^{4}$ cells $/ \mathrm{cm}^{2}$ for experiments. Low glucose condition describes cells cultured in medium with $5 \mathrm{mM}$ glucose. Pyrollidine dithiocarbamate, an NF-KB inhibitor, was used to inhibit NF-kB signaling at various concentrations.

\section{Immunofluorescence}

BV2 microglia were fixed with 4\% paraformaldehyde at room temperature, permeabilized for $5 \mathrm{~min}$ with $0.1 \%$ Triton-X (Sigma) and blocked with 1\% BSA in PBST for $1 \mathrm{~h}$. Then, cells were incubated with primary antibodies in $1 \%$ BSA overnight at $4^{\circ} \mathrm{C}, 1 \mathrm{~h}$ the next day with secondary antibodies (Jackeson, 1:1000) and then with DAPI for $30 \mathrm{~min}$ at room temperature. The primary antibodies included mouse anti-MCT1 ( ab10582, abcom, 1:250)『rabbit anti-argnase-1(sc-166902, Santa Cruz, 1:200) \goat anti-Iba1(MABN92, Novus, 1:2000), rabbit anti-iNOS (\#13120,CST, 1:1000). Cells were evaluated under 40x magnification using a Vision microscope (Olympus, Japan). All imaging conditions, including exposure times and image acquisition, were identical.

Brain tissue samples were harvested and $10 \mu \mathrm{m}$-thick cryosections were generated using a microtome (Leica CM1950). Sections were incubated in citrate buffer for antigen recovery, then in $0.3 \%$ hydrogen peroxide for $20 \mathrm{~min}$, and blocked with blocking buffer for $1 \mathrm{~h}$. Similarly, sections were incubated with primary and secondary antibodies as described above and processed using the fluorescence microscope (Olympus, Japan).

\section{Western blotting}

$20 \mathrm{ng}$ of protein lysates were loaded on 10\% SDS polyacrylamide gel for protein separation and transferred to a polyvinylidene difluoride membrane. After blocking with $5 \%$ nonfat milk for $1 \mathrm{~h}$, membranes were incubated with primary antibodies at $4{ }^{\circ} \mathrm{C}$ overnight and rinsed with tris buffered saline tween (TBST) buffer before incubation with secondary antibodies for 1 hour. Membranes were imaged using Tanon-4500 after addition of peroxidase reagents. 


\section{MCT1 knockdown}

All small interfering RNAs were purchased from MICRO-G BIOTECK company (Shanghai, China). According to the manufacture's protocol, BV2 microglia were seeded at $10^{5}$ cells/well. $20 \mu \mathrm{M}$ of siRNA was added to serum-free DMEM and then mixed with $5 \mu$ of Lipo3000 transfection reagent. After incubation at room temperature for $15 \mathrm{~min}$, the mixture was added to cells and cultured for $48 \mathrm{~h}$. The sequences of siRNAs were as follows (5'-3'): forward UUCUCCGAACGUGUCACGUTT, reverse ACGUGACACGUUCGGAGAATT for siRNA-control; forward CCAAAUCCAUCACUGUCUUTT, reverse AAGACAGUGAUGGAUUUGG for siRNA-MCT1.

\section{MCT1-containing plasmid transfection}

The GV230 eukaryotic plasmids were purchased from TSINGKE BIOTECK (Tianjin, China). First, Xhol/Kpnl double enzyme digestion was performed on plasmids for MCT1 gene insertion. For transfection, microglia were grown in Dulbecco's Modified Eagle's Medium(Gibco), supplemented with 10\% FBS (Gibco) and 1\% penicillin/streptomycin (Gibco) in a 5\% CO2 incubator at $37^{\circ} \mathrm{C}$. Appropriate

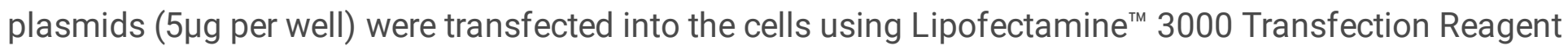
(L3000-015, Invitrogen) according to the manufacturer's instructions.

\section{Transmission electron microscopy}

BV2 microglia were harvested with cell scrapper and fixed with $2.5 \%$ glutaric dialdehyde for $2 \mathrm{~h}$ at $4^{\circ} \mathrm{C}$, rinsed with $0.1 \mathrm{M}$ of phosphoric acid, and fixed again with osmic acid for $3 \mathrm{~h}$. Cells were further incubated in $1 \%$ uranyl acetate for $2 \mathrm{~h}$. After dehydration in acetone, cells were dried at $65^{\circ} \mathrm{C}$ for $48 \mathrm{~h}$. Cells were analyzed with transmission electron microscope (h7500, HITACHI).

\section{Statistical analysis}

For western blotting, triplicates were performed for three independent experiments. For immunofluorescence imaging, 3 random views of each group were selected and at least 100 cells were counted. All data were analyzed by one-way ANOVA and Tukey post hoc test. $p<0.05$ was viewed as statistically significant.

\section{Results}

\section{Activated microglia express iNOS, ARG1 and MCT1 in laser-induced ischemic mouse brains}

The infarct areas were confirmed with TTC staining in laser-induced ischemic mouse brains. (Figure 1A). Compared to the control group, iNOS and ARG1 protein expressions were both increased in the ischemic brains (Figure 1B). Immunofluorescence staining showed that the expressions of Iba1, MCT, iNOS and ARG1 increased after cerebral ischemia (Figure1C, D). Immunofluorescence double labeling showed that Iba1 and iNOS immunopositive co-localization was significantly more than Iba1 and ARG1 colocalization, and MCT1 was mainly expressed in iNOS positive microglia (Figure 1E). 


\section{Increased expression of MCT1 in BV2 cells and primary microglia under low glucose condition}

BV2 microglia were placed in low glucose medium, and iNOS expression increased while ARG1 expression decreased over a 2-hour period. The expression of MCT1 was found upregulated and mainly localized with iNOS ${ }^{+}$and less with ARG1 ${ }^{+}$BV2 microglia (Figure 2A). The same was observed in primary microglia cultured in low-glucose medium, where expressions of iNOS and MCT1 rose drastically, while ARG1 declined. In addition, CD16/32 also increased, suggesting M1 polarization was successful after 2 hours of low glucose treatment (Figure $3 \mathrm{~A}$ ). Upregulation of MCT1 and iNOS and downregulation of ARG1 were further confirmed by western blot in both BV2 and primary microglia (Figure 2B, Figure 3B). These data demonstrated that microglia polarize to the M1 phenotype accompanied by increase of MCT1 under low glucose condition.

\section{Pyruvate sustains the M1 phenotype in primary microglia}

Pyruvate, a monocarboxylate, is the end-product of glycolysis and may be utilized by microglia when glucose supply is low. To test this, we first cultured primary microglia with either LPS or low-glucose medium. Polarization of microglia was confirmed by increased expression of iNOS and decreased expression of ARG1. Then, we supplemented the medium with sodium pyruvate (SP), the amplitudes of change further increased with a statistics significance for both iNOS and ARG1 (Figure 4A). Since pyruvate is metabolized in the mitochondria, we utilized transmission electron microscopy and found that in low-glucose group, the number of mitochondria and the variation in mitochondrial diameter were changed, but not obviously than those in control group. After the addition of sodium pyruvate, these changes were significantly more pronounced (Figure 4B). In the LPS-treated group, there is a large variance in mitochondrial diameter similar to that observed in the low glycose group with sodium pyruvate, but it did not affect mitochondrial number.

\section{NF-KB regulates expressions of MCT1 and iNOS}

We have demonstrated that pyruvate can sustain iNOS production in microglia. Next, we investigated whether MCT1 can regulate iNOS production. Overexpression and knockdown of MCT1 were confirmed by western blot (Figure 5). The expression of iNOS positively correlated with MCT1, in which iNOS increased upon MCT1 overexpression and decreased after MCT1 knockdown (Figure 5). The addition of NF-KB inhibitor, Pyrollidine dithiocarbamate (Pdb), was found to reduce the expression of both MCT1 and iNOS (Figure 6). ARG1 expression, on the other hand, was not affected by MCT1 manipulation (Figure 5) nor by NF-KB inhibitor (Figure 6). These data have shown that MCT1 can influence iNOS production and is regulated by the NF-KB signaling pathway in low glucose-activated M1 microglia.

\section{Discussion}

Microglia adopt different activated states and perform multiple functions to confine and reduce tissue damage and restore CNS function. A switch from oxidative phosphorylation in the mitochondria to anaerobic glycolysis in the cytoplasm has been repeatedly demonstrated in M1 macrophages[27]. 
Although glucose is the preferred energy substrate by microglia for ATP production, its availability is significantly reduced during cerebral ischemia. Ischemic brain region is characterized by hypoxia and high levels of lactate and $\mathrm{H}^{+}$[46]. Under such environment, alternative energy sources have not been carefully evaluated. Here, we used the laser-induced ischemic mouse model to examine the underlying reasons behind sustained expressions of iNOS and MCT1 in damaged rodent brains. Previously, increase in MCT1 expression was reported after ischemia in mice[47, 48]. We demonstrated that microglia produced greater iNOS ${ }^{+}$and MCT1 under low glucose condition. We suspect that within ischemic area, microglia require more MCT1 to sustain iNOS production. It is conceivable that more monocarboxylates are transported into the mitochondria through $\mathrm{MCT} 1 . \mathrm{ARG} 1^{+}$microglia have also been detected but less of them co-localize with MCT1.

We also detected increased iNOS and MCT1 in the BV2 cell line and primary microglia after low glucose treatment, which are consistent with our in vivo data. One notable difference was decreased ARG1 expression under low glucose treatment, which may be attributed to the variable quiescent states of cultured cells. Regular culture medium contains $25 \mathrm{mM} / \mathrm{L}$ of glucose, as compared to a five-fold reduction to $5 \mathrm{mM} / \mathrm{L}$ in the low glucose medium used in this study. Low glucose condition would result in a shift in NADH:NAD ${ }^{+}$ratio towards $\mathrm{NADH}$ within the cytoplasm and promote pro-inflammatory gene expression through the NF-KB pathway [26].

In primary microglia, addition of pyruvate after low glucose treatment enhanced iNOS expression, which was also observed in the LPS-treated group. Changes in mitochondrial number and morphology under low-glucose condition supplemented with pyruvate suggested that mitochondria were involved in the M1 microglial phenotype $[49,50]$. Although mitochondrial diameters also varied considerably under LPS stimulation, the presence of autophagosomes implied that mitochondria may have undergone fission as LPS is a stronger stimulus than reduced glucose. These data demonstrated that exogenous pyruvate can be used to generate ATP and continue to maintain the M1 phenotype. Therefore, under ischemia, it is possible that lactate, another monocarboxylate, is taken up by the highly expressed MCT1 and converted to pyruvate for use in the mitochondria.

NF-KB is vital in low glucose-induced microglial activation [51] and for iNOS [52, 53] and MCT1[54] expressions. But, the relationship between MCT1 and iNOS has not been explored. Here, MCT1 knockdown significantly reduced expression of iNOS. The opposite was seen under overexpression of MCT1, where iNOS was increased. The addition of NF-KB inhibitor led to reduced MCT1 and iNOS in a concentration-dependent manner. These results suggested that MCT1 is upstream of iNOS, and both can be regulated by the NF-KB signaling pathway.

\section{Conclusions}

Our study on mouse model with laser-induced brain ischemia and cell culture with low-glucose treatment demonstrated that glucose shortage induces proinflammatory microglia activation and promotes the expression of MCT1 and iNOS through NF-KB regulation. MCT1 allows the uptake of pyruvate, which may 
be utilized by the mitochondria to generate ATP and sustain iNOS production under the inflammatory response.

\section{Abbreviations}

iNOS: Inducible nitric oxide synthase; MCT1: Monocarboxylate transporter 1; ARG1: Arginase-1; LPS: Lipopolysaccharide; NF-kB: Nuclear factor-kappaB; CNS: Central nervous system; TTC: 2,3,5-triphenyl tetrazolium chloride; GM-CSF: Granulocyte-macrophage colony-stimulating factor; TBST: Tris buffered saline tween

\section{Declarations}

\section{Acknowledgements}

Not applicable.

\section{Authors' contributions}

ML was involved in project design and data analysis; JJD, SCY, CHR performed the major experimental work; YCX, MCD wrote the paper; YCX revised the manuscript. All authors approved the final manuscript before submission.

\section{Funding}

This study is supported by Natural Science of the Zhejiang Province Foundation of China [Grant numbers: LY19H090009, LY20H090010].

\section{Conflict of interest}

None

\section{Availability of data and materials}

The manuscript included all relevant data. 


\section{Ethics approval and consent to participate}

All animal procedures were approved by the Animal Research Ethics Committee of China Wenzhou Medical University (wydw2019-0839).

\section{Consent for publication}

Not applicable.

\section{Competing interests}

The authors declare that they have no competing interests.

\section{References}

1. Wolf SA, Boddeke HW, Kettenmann H. Microglia in Physiology and Disease. Annu Rev Physiol. 2017;79:619-43.

2. Kawabori M, Yenari MA. The role of the microglia in acute CNS injury. Metab Brain Dis. 2015;30:38192.

3. Lloyd AF, Davies CL, Holloway RK, Labrak Y, Ireland G, Carradori D, Dillenburg A, Borger E, Soong D, Richardson JC, Kuhlmann T, Williams A, Pollard JW, A des Rieux, Priller J, Miron VE, Central nervous system regeneration is driven by microglia necroptosis and repopulation, Nature neuroscience, 22 (2019) 1046-1052.

4. Seney ML, Huo Z, Cahill K, French L, Puralewski R, Zhang J, Logan RW, Tseng G, Lewis DA, Sibille E. Opposite Molecular Signatures of Depression in Men and Women, Biological psychiatry, 84 (2018) 18-27.

5. Cathomas F, Azzinnari D, Bergamini G, Sigrist H, Buerge M, Hoop V, Wicki B, Goetze L, Soares S, Kukelova D, Seifritz E, Goebbels S, Nave K-A, Ghandour MS, Seoighe C, Hildebrandt T, Leparc G, Klein $\mathrm{H}$, Stupka E, Hengerer B, Pryce CR. Oligodendrocyte gene expression is reduced by and influences effects of chronic social stress in mice. Genes Brain Behav. 2019;18(1):e12475.

6. Kemal Shahrnaz., Vassar Robert. Death by microglia. J Exp Med. 2019;216(11):2451-2.

7. Dorman LC, Molofsky AV, Demystifying Microglia: And Now the Work Begins, Immunity, 50 (2019) 11-13.

8. Collmann FM, Pijnenburg R, Hamzei-Taj S, Minassian A, Folz-Donahue K, Kukat C, Aswendt M, Hoehn $M$, Individual in vivo Profiles of Microglia Polarization After Stroke, Represented by the Genes iNOS and Ym1, Frontiers in immunology, 10 (2019) 1236. 
9. Liddelow Shane A, Guttenplan Kevin A, Clarke Laura E, Bennett Frederick C, Bohlen Christopher J, Lucas S., Bennett Mariko L, Münch Alexandra E, Chung Won-Suk., Peterson Todd C, Wilton Daniel K, F Arnaud., Napier Brooke, APanicker Nikhil., K Manoj., Buckwalter Marion, Rowitch David S, Dawson Valina H, L, Dawson Ted, M, Stevens Beth., B Ben A.(2017). Neurotoxic reactive astrocytes are induced by activated microglia. Nature, 541(7638), 481-487.

10. Kucharova K, Stallcup WB. Dissecting the multifactorial nature of demyelinating disease. Neural regeneration research. 2018;13:628-32.

11. Yang Zhiyou., Kuboyama Tomoharu., Tohda Chihiro.(2019). Naringenin promotes microglial M2 polarization and $A \beta$ degradation enzyme expression. Phytother Res, 33(4), 1114-1121.

12. Ghosh $M, X u Y, P e a r s e ~ D D$. Cyclic AMP is a key regulator of $M 1$ to M2a phenotypic conversion of microglia in the presence of Th2 cytokines. J Neuroinflamm. 2016;13:9.

13. Beckmann N, Giorgetti E, Neuhaus A, Zurbruegg S, Accart N, Smith P, Perdoux J, Perrot L, Nash M, Desrayaud S, Wipfli P, Frieauff W, Shimshek DR. Brain region-specific enhancement of remyelination and prevention of demyelination by the CSF1R kinase inhibitor BLZ945, Acta neuropathologica communications, 6 (2018) 9.

14. Ogawa Y, Irisa M, Sano T, Yanagi Y, Furusawa E, Saito T, Yamanaka S, Itoh K, Sakuraba H, Oishi K. Improvement in dysmyelination by the inhibition of microglial activation in a mouse model of Sandhoff disease. Neuroreport. 2018;29:962-7.

15. Wu Yuwen

Wu Yuwen., Dissing-Olesen Lasse., MacVicar Brian A, Beth S.(2015). Microglia: Dynamic Mediators of Synapse Development and Plasticity. Trends Immunol., 36(10), 605-613.

16. Jiang T, Zhang L, Pan X, Zheng H, Chen X, Li L, Luo J, Hu X. Physical Exercise Improves Cognitive Function Together with Microglia Phenotype Modulation and Remyelination in Chronic Cerebral Hypoperfusion. Frontiers in cellular neuroscience. 2017;11:404.

17. Colonna Marco., Butovsky Oleg. Microglia Function in the Central Nervous System During Health and Neurodegeneration. Annu Rev Immunol. 2017;35(undefined):441-68.

18. Yeh FL, Hansen DV, Sheng M. TREM2, Microglia, and Neurodegenerative Diseases. Trends Mol Med. 2017;23:512-33.

19. Kierdorf K, Prinz M. Microglia: Same same, but different. J Exp Med. 2019;216:2223-5.

20. Tang Y, Le W. Differential Roles of M1 and M2 Microglia in Neurodegenerative Diseases. Mol Neurobiol. 2016;53:1181-94.

21. Loane DJ, Kumar A. Microglia in the TBI brain: The good, the bad, and the dysregulated, Experimental neurology. 275 Pt. 2016;3:316-27.

22. Kalsbeek MJ, Mulder L, Yi CX. Microglia energy metabolism in metabolic disorder. Mol Cell Endocrinol. 2016;438:27-35.

23. Jha MKM. B. M., Glia-neuron energy metabolism in health and diseases: New insights into the role of nervous system metabolic transporters. Exp Neurol. 2018;309:23-31. 
24. Zhao B, Wang H, Li CX, Song SW, Fang SH, Wei EQ, Shi QJ. GPR17 mediates ischemia-like neuronal injury via microglial activation. Int J Mol Med. 2018;42:2750-62.

25. Shen Y, Kapfhamer D, Minnella AM, Kim JE, Won SJ, Chen Y, Huang Y, Low LH, Massa SM, Swanson RA. Bioenergetic state regulates innate inflammatory responses through the transcriptional corepressor CtBP. Nature communications. 2017;8:624.

26. Ghosh S, Castillo E, Frias ES, Swanson RA. Bioenergetic regulation of microglia. Glia. 2018;66:120012.

27. Galván-Peña, Silvia O'Neill, Luke A. \& J. Metabolic reprograming in macrophage polarization. Frontiers in Immunology. 2014;5:420.

28. Vegran F, Boidot R, Michiels C, Sonveaux P, Feron O. Lactate influx through the endothelial cell monocarboxylate transporter MCT1 supports an NF-kappaB/IL-8 pathway that drives tumor angiogenesis. Cancer research. 2011;71:2550-60.

29. 173

A Universal Immune Sensor of Neurodegeneration

Disease-Associated Microglia

1073

1081

Deczkowska A, Keren-Shaul H, Weiner A, Colonna M, Schwartz M, Amit I. Disease-Associated Microglia: A Universal Immune Sensor of Neurodegeneration, Cell, 173 (2018) 1073-1081.

30. Salter MW, Stevens B. Microglia emerge as central players in brain disease. Nature medicine. 2017;23:1018-27.

31. Kierdorf K, Prinz M. Microglia in steady state. J Clin Investig. 2017;127:3201-9.

32. Brooks GA, Brown MA, Butz CE, Sicurello JP, Dubouchaud H. Cardiac and skeletal muscle mitochondria have a monocarboxylate transporter MCT1. J. Appl. Physiol., 87(5)(1999), 1713-8.

33. Hashimoto T, Hussien R, Cho HS, Kaufer D, Brooks GA. Evidence for the mitochondrial lactate oxidation complex in rat neurons: demonstration of an essential component of brain lactate shuttles. PloS one. 2008;3:e2915.

34. Moreira TJ, Pierre K, Maekawa F, Repond C, Cebere A, Liljequist S, Pellerin L. Enhanced cerebral expression of MCT1 and MCT2 in a rat ischemia model occurs in activated microglial cells. Journal of cerebral blood flow metabolism: official journal of the International Society of Cerebral Blood Flow Metabolism. 2009;29:1273-83.

35. Kong L, Wang Z, Liang X, Wang Y, Ma C.. . Monocarboxylate transporter 1 promotes classical microglial activation and pro-inflammatory effect via 6-phosphofructo-2-kinase/fructose-2, 6biphosphatase 3. Journal of Neuroinflammation, 16(1)(2019).

36. Chong CM, Ai N, Ke M, Tan Y, Huang Z, Li Y, Lu JH, Ge W, Su H, Roles of Nitric Oxide Synthase Isoforms in Neurogenesis, Molecular neurobiology, 55 (2018) 2645-2652.

37. Maksoud MJE, Tellios V, An D, Xiang YY, Lu WY. Nitric oxide upregulates microglia phagocytosis and increases transient receptor potential vanilloid type 2 channel expression on the plasma membrane. 
Glia. 2019;67:2294-311.

38. Lisi L, Ciotti GM, Braun D, Kalinin S, Curro D, Dello Russo C, Coli A, Mangiola A, Anile C, Feinstein DL, Navarra P. Expression of iNOS, CD163 and ARG-1 taken as M1 and M2 markers of microglial polarization in human glioblastoma and the surrounding normal parenchyma. Neurosci Lett. 2017;645:106-12.

39. Sonar SA, Lal G. The iNOS Activity During an Immune Response Controls the CNS Pathology in Experimental Autoimmune Encephalomyelitis. Frontiers in immunology. 2019;10:710.

40. Kim ME, Jung I, Na JY, Lee Y, Lee J, Lee JS, Lee JS, Pseudane-VII Regulates LPS-Induced Neuroinflammation in Brain Microglia Cells through the Inhibition of iNOS Expression M. (Basel, Switzerland), 23 (2018).

41. Pereira-Vieira JA-S, Preto J, Casal A, Queiros M. O., MCT1, MCT4 and CD147 expression and 3bromopyruvate toxicity in colorectal cancer cells are modulated by the extracellular conditions. Biol Chem. 2019;400:787-99.

42. Yue Y, Stone S, Lin W. Role of nuclear factor kappaB in multiple sclerosis and experimental autoimmune encephalomyelitis. Neural regeneration research. 2018;13:1507-15.

43. Chen J, Sun Z, Jin M, Tu Y, Wang S, Yang X, Chen Q, Zhang X, Han Y, Pi R. Inhibition of AGEs/RAGE/Rho/ROCK pathway suppresses non-specific neuroinflammation by regulating BV2 microglial M1/M2 polarization through the NF-kappaB pathway. J Neuroimmunol. 2017;305:108-14.

44. Huang C, Lu X, Wang JL, Tong LJ, Ling Y, Jiang B, Yang RR, Zhang W. Compound C induces the ramification of murine microglia in an AMPK-independent and small rhogtpase-dependent manner. Neuroscience. 2016;331:24-39.

45. Peng W. Neuroprotective effects of G-CSF administration in microglia-mediated reactive $T$ cell activation in vitro. Immunol Res. 2017;65:888-902.

46. Fann DY, Santro T, Manzanero S, Widiapradja A, Cheng YL, Lee SY, Chunduri P, Jo DG, Stranahan AM, Mattson MP, Arumugam TV. Intermittent fasting attenuates inflammasome activity in ischemic stroke. Exp Neurol. 2014;257:114-9.

47. Zhou PG, Jiang T, Namaka Z, Huang M, Kong QJ. J. M., Monocarboxylate transporter 1 and the vulnerability of oligodendrocyte lineage cells to metabolic stresses. CNS Neurosci Ther. 2018;24:126-34.

48. Fisel P,Schaeffeler Elke,Schwab Matthias,Clinical and Functional Relevance of the Monocarboxylate Transporter Family in Disease Pathophysiology and Drug Therapy.[J].Clin Transl Sci, 2018, 11: 352364.

49. Owens K, Park JH, Schuh R, Kristian T. Mitochondrial dysfunction and NAD(+) metabolism alterations in the pathophysiology of acute brain injury. Translational stroke research. 2013;4:61834.

50. Jensen MB, Jasper $\mathrm{H}$. Mitochondrial proteostasis in the control of aging and longevity. Cell Metabol. 2014;20:214-25. 
51. Huang Wen B, Shunjie Z, Xuzheng T, Weiju C Pengfei., C Xiuying., WGong,W Haoxiang., X Peng. An adenosine A1R-A2aR imbalance regulates low glucose/hypoxia-induced microglial activation, thereby contributing to oligodendrocyte damage through NF-KB and CREB phosphorylation. Int. J. Mol. Med., 41(6)(2018)3559-3569.

52. Soraya Abbasi FS, Habashi*1. Ali Moghimi1 and Saeed Ansari Majd2, Modulation of Lipopolysaccharide Stimulated Nuclear Factor kappa B Mediated iNOS/NO Production by Bromelain in Rat Primary Microglial Cells, Iranian Biomedical Journal, (2016).

53. Kleinert H, Euchenhofer C, Ihrig-Biedert I, Förstermann U. Glucocorticoids inhibit the induction of nitric oxide synthase II by down-regulating cytokine-induced activity of transcription factor nuclear factorkappa B. Mol Pharmacol. 1996;49:15-21.

54. Hashimoto T, Hussien R, Oommen S, Gohil K, Brooks GA. Lactate sensitive transcription factor network in L6 cells: activation of MCT1 and mitochondrial biogenesis. FASEB J. 2007;21:2602-12.

\section{Figures}




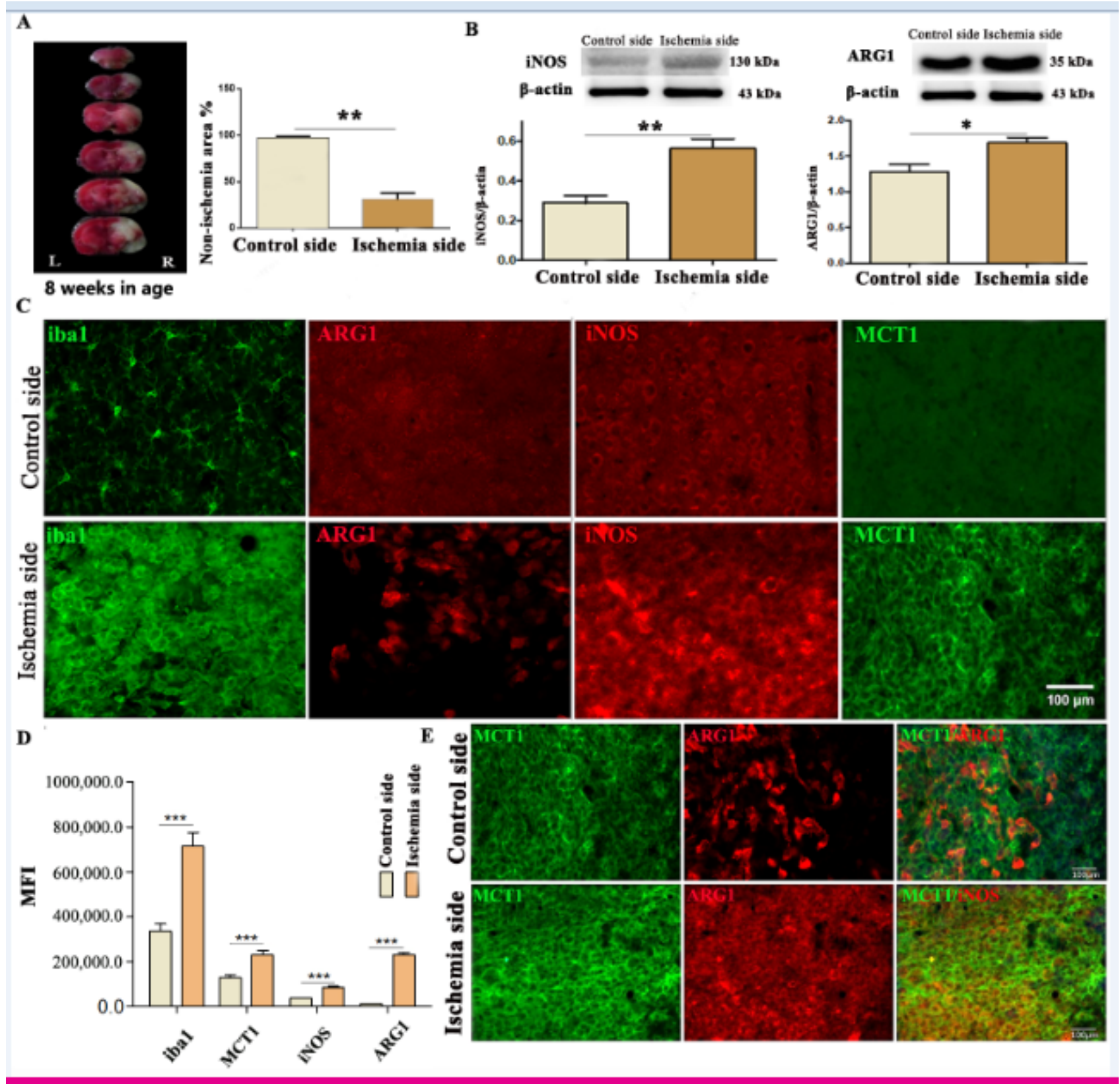

Figure 1

[No figure caption provided with this version.] 


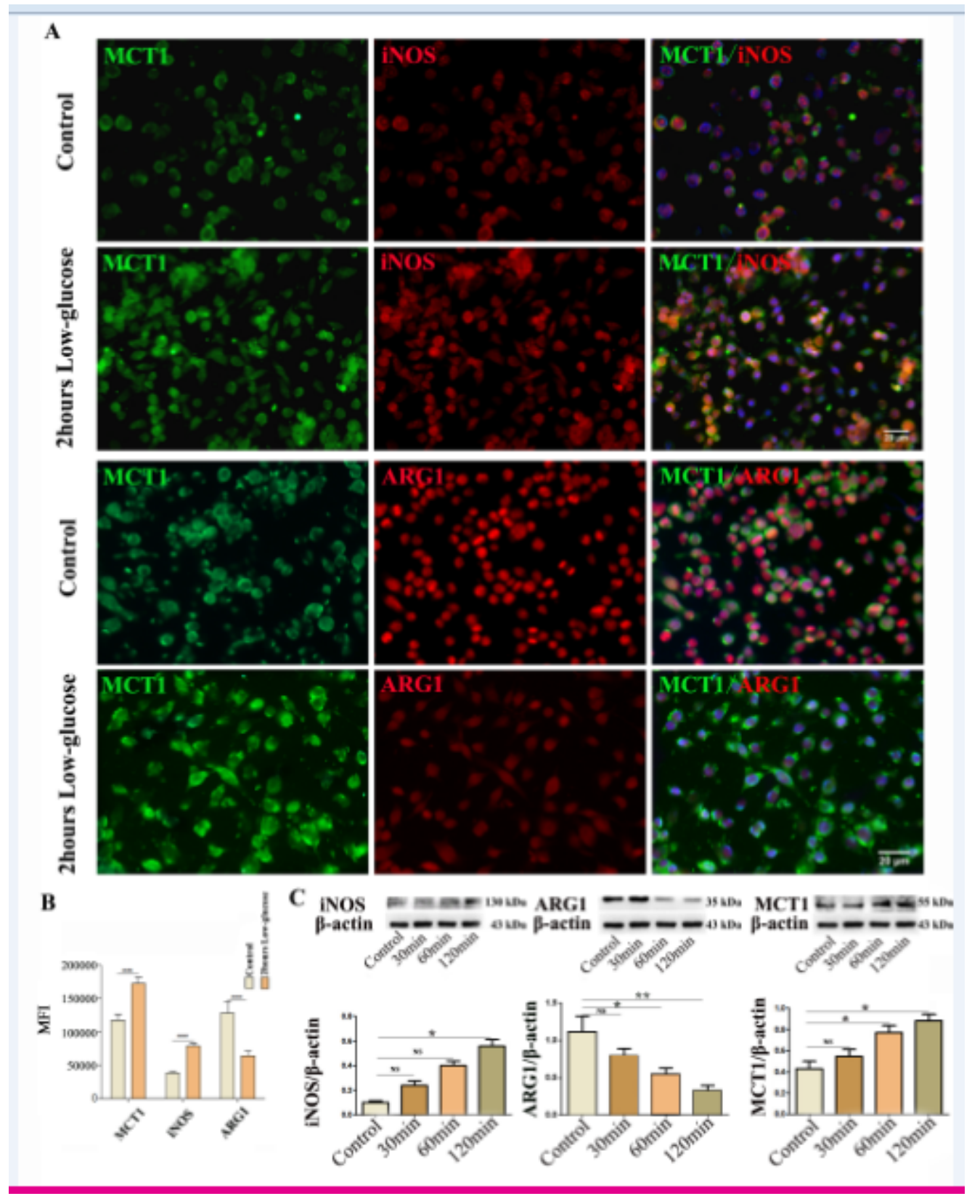

Figure 2

[No figure caption provided with this version.] 


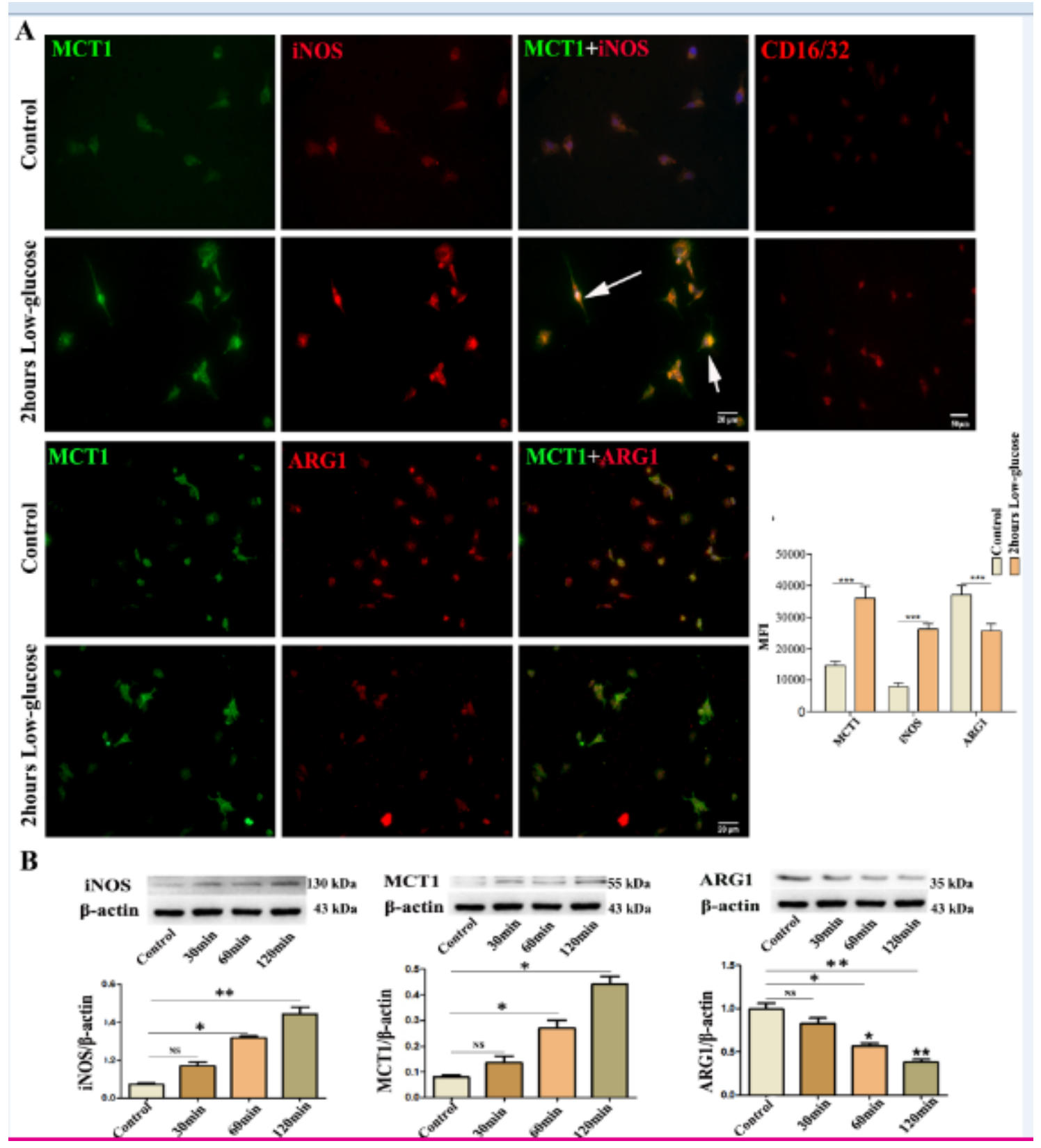

Figure 3

[No figure caption provided with this version.] 


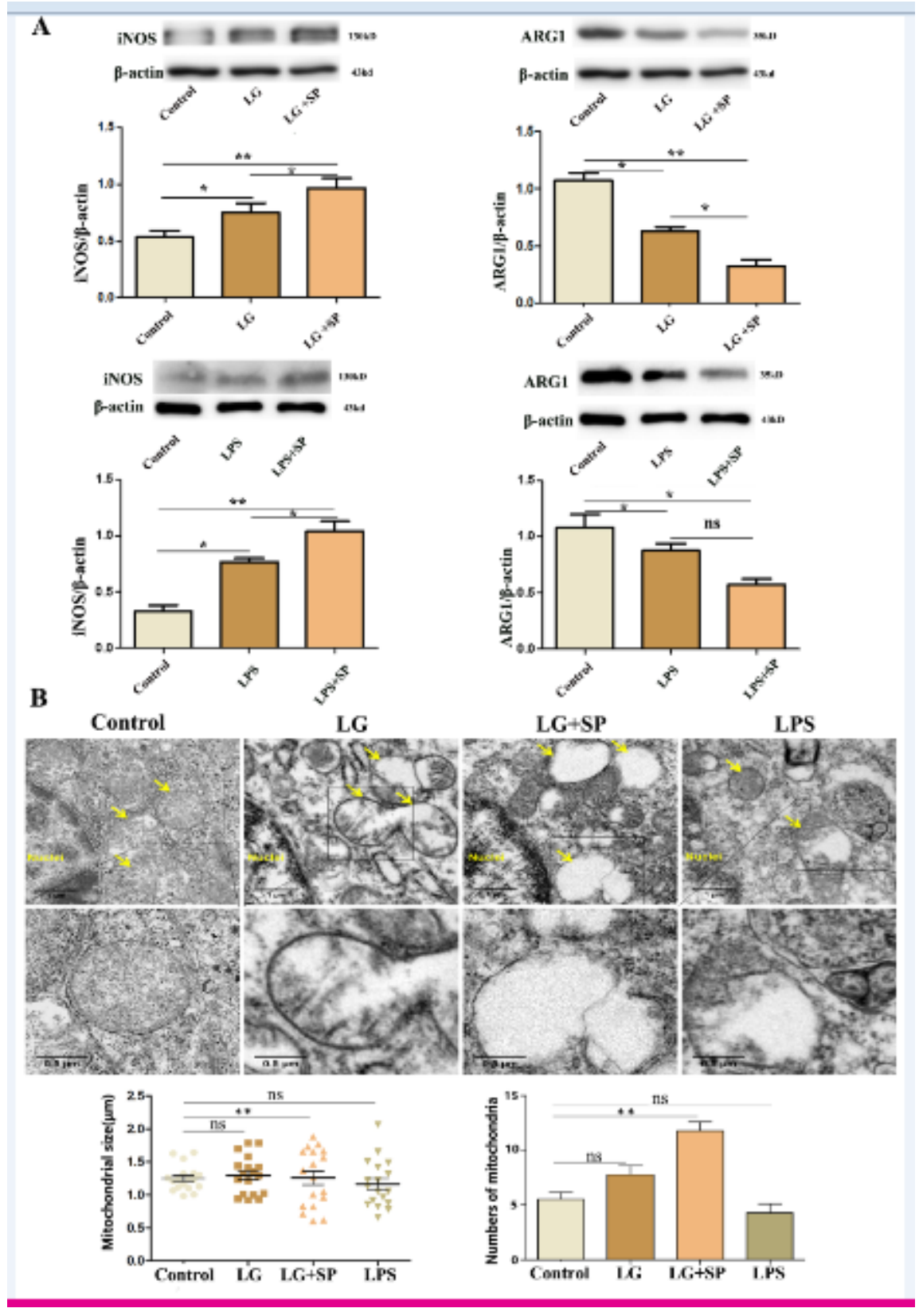

Figure 4

[No figure caption provided with this version.] 


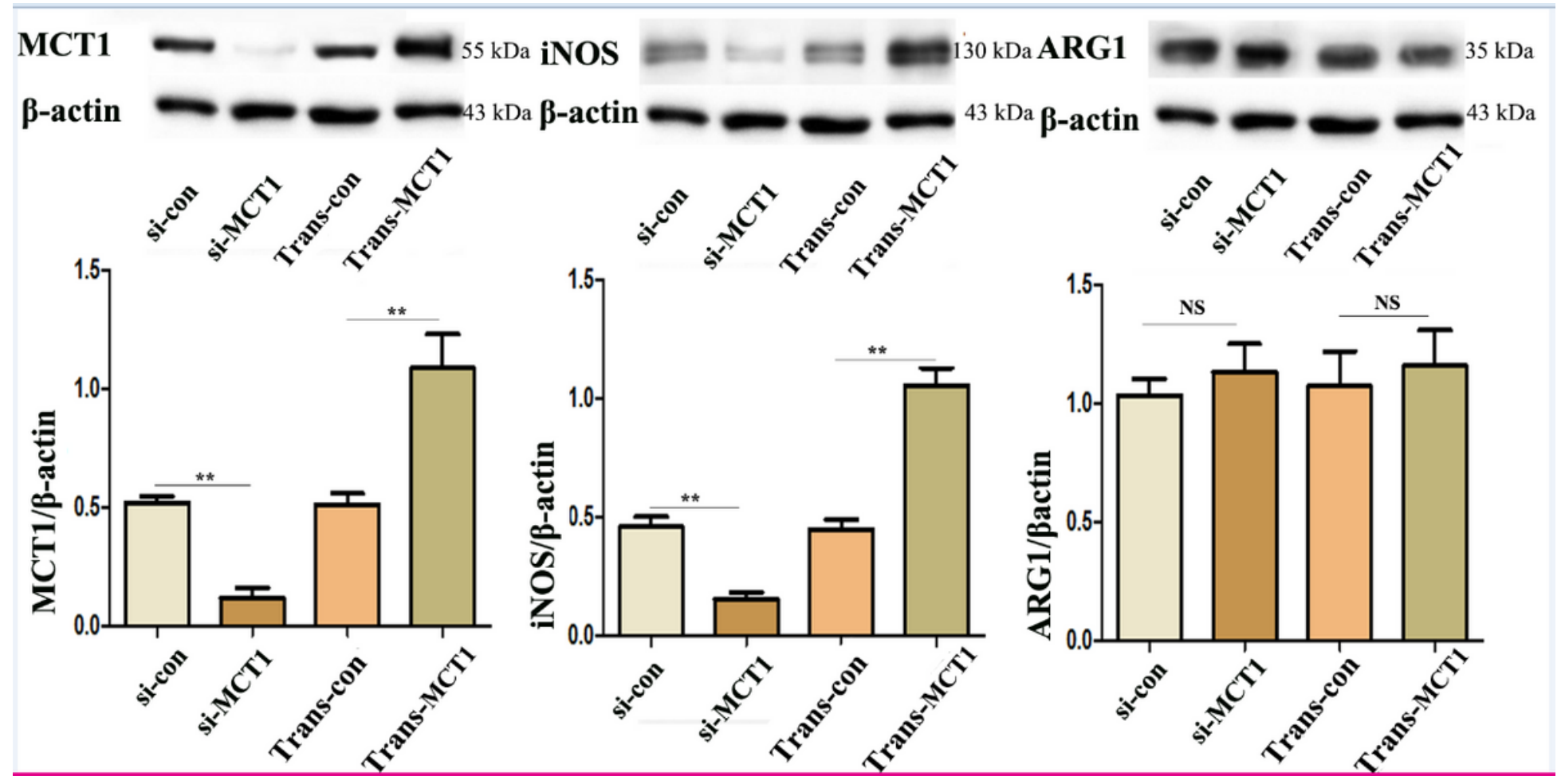

Figure 5

Overexpression and knockdown of MCT1 were confirmed by western blot.

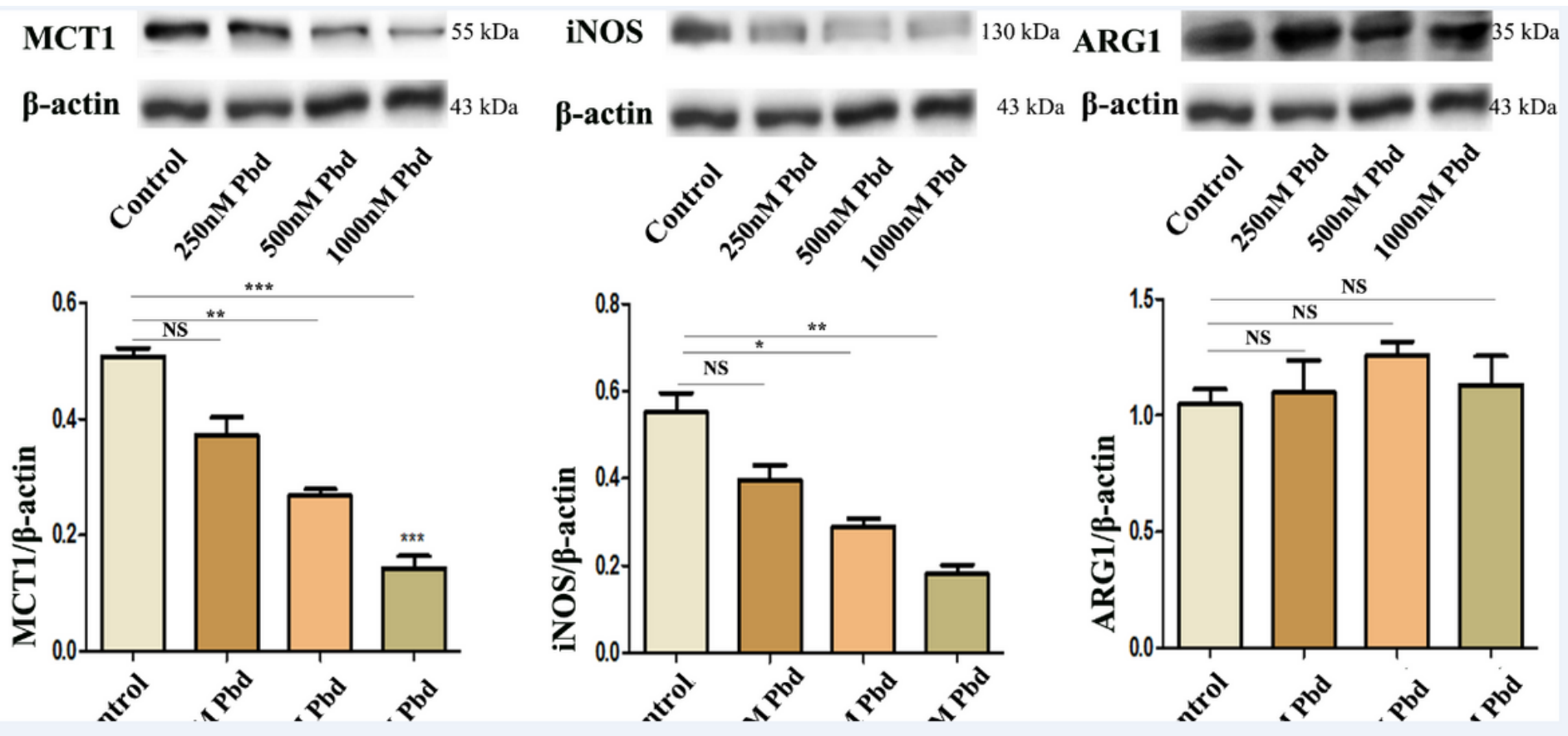

Figure 6

The addition of NF-KB inhibitor, Pyrollidine dithiocarbamate (Pdb), was found to reduce the expression of both MCT1 and iNOS. 